This article was downloaded by: [Goldsmiths, University of London]

On: 28 January 2014, At: 01:43

Publisher: Routledge

Informa Ltd Registered in England and Wales Registered Number: 1072954 Registered office: Mortimer House, 37-41 Mortimer Street, London W1T 3J H, UK

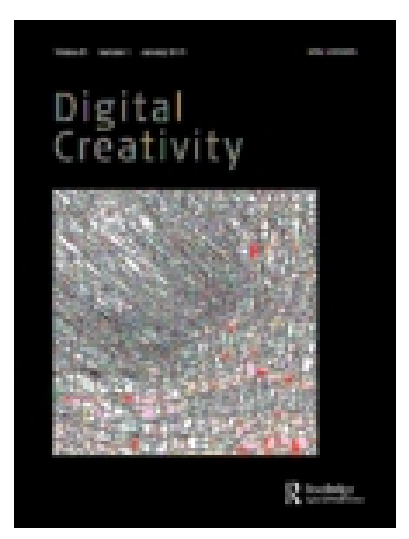

\title{
Digital Creativity
}

Publication details, including instructions for authors and subscription information: http:// www. tandfonline.com/loi/ndcr20

\section{Net Work: an interactive artwork designed using an interdisciplinary performative approach}

\author{
J on Bird ${ }^{a}$, Mark D'inverno ${ }^{b} \&$ J ane Prophet ${ }^{c}$ \\ ${ }^{a}$ University of Sussex, UK E-mail: \\ ${ }^{b}$ Goldsmiths College, University of London, UK E-mail: \\ ${ }^{\mathrm{c}}$ Independent artist, UK E-mail: \\ Published online: 20 Mar 2007.
}

To cite this article: J on Bird, Mark D'inverno \& J ane Prophet (2007) Net Work: an interactive artwork designed using an interdisciplinary performative approach, Digital Creativity, 18:1, 11-23, DOI: 10.1080/14626260701252368

To link to this article: http:// dx. doi. org/ 10.1080/ 14626260701252368

\section{PLEASE SCROLL DOWN FOR ARTICLE}

Taylor \& Francis makes every effort to ensure the accuracy of all the information (the "Content") contained in the publications on our platform. However, Taylor \& Francis, our agents, and our licensors make no representations or warranties whatsoever as to the accuracy, completeness, or suitability for any purpose of the Content. Any opinions and views expressed in this publication are the opinions and views of the authors, and are not the views of or endorsed by Taylor \& Francis. The accuracy of the Content should not be relied upon and should be independently verified with primary sources of information. Taylor and Francis shall not be liable for any losses, actions, claims, proceedings, demands, costs, expenses, damages, and other liabilities whatsoever or howsoever caused arising directly or indirectly in connection with, in relation to or arising out of the use of the Content.

This article may be used for research, teaching, and private study purposes. Any substantial or systematic reproduction, redistribution, reselling, loan, sub-licensing, systematic supply, or distribution in any form to anyone is expressly forbidden. Terms \& Conditions of access and use can be found at http://www.tandfonline.com/page/termsand-conditions 


\title{
Net Work: an interactive artwork designed using an interdisciplinary performative approach
}

\author{
Jon Bird ${ }^{1}$, Mark d'Inverno ${ }^{2}$ and Jane Prophet ${ }^{3}$ \\ 1 University of Sussex, UK \\ jonba@sussex.ac.uk \\ 2 Goldsmiths College, University of London, UK \\ dinverno@gold.ac.uk \\ 3 Independent artist, UK \\ jane@janeprophet.com
}

\begin{abstract}
In this paper we outline an interdisciplinary collaborative approach to problem solving that can be characterised as performative as both the goals and solutions develop over time through an open-ended process of trial-and-error. We describe two projects where this methodology has been successfully applied. We first give an overview of the CELL project where the performative approach led to a significant change in the way that scientist Neil Theise investigated stem cells. The success of this project directly led to the work which is the main focus of this paper: the design of Net Work, an interactive artwork that consists of a grid of autonomous buoys that emit different coloured light in response to the environment and the state of neighbouring buoys. We describe our performative approach to building the Net Work prototype and outline in detail its control system which is based on Ashby's homeostat model. The paper concludes with a short discussion of some of the benefits and pitfalls of an interdisciplinary collaborative approach to problem solving.
\end{abstract}

Keywords: interactive artwork, homeostat, interdisciplinary and performative research, multi-agent systems, problem solving

\section{Introduction}

The whole point about the performative idiom... [is] to see the world as a lively place full of agency - not something static and dead, sitting around waiting to be represented, as the representational idiom suggests. And it now strikes me that the homeostat is a very nice device to illustrate that ontology. The homeostat was visibly an electromechanical device situated in the material world, but it was a surprising one-one could never tell from the outside how it would reconfigure itself next, what it would do next. And one can go further than that. In the four-homeostat set-up, imagine one of the units as standing for some organic being in the world - a scientist say - and the other three units as standing for that being's material environment. Then the scientist-homeostat could be seen as openendedly searching through spaces of the material performativity of its environment, which itself open-endedly and unpredictably unfolds, with the final stable state of the four-homeostat assemblage appearing as the emergent joint product of open-ended trial and error through states of both the scientist and the environment.

(Pickering 2002, p.416) 
The term 'performative' is applied to diverse activities, ranging from science to curation, and it is used to signify "the constitution of meaning through an act or a certain practice" (Schlieben 2002). The term originates from the philosophy of language where it describes speech acts, such as 'I swear' or 'I bet', which perform, rather than describe, the action mentioned (Austin 1962). In this paper we describe a collaborative interdisciplinary approach to problem solving that can be characterized as performative. We describe its application in two projects: $C E L L$, which investigated stem cell behaviour; and an EPSRC/AHRC funded interdisciplinary research cluster (IRC) that focused on new forms of design process suitable for the challenges of the $21^{\text {st }}$ century and produced a prototype of Net Work, an interactive artwork. Both projects did not begin with any clearly defined collective goals; instead, the goals, and their solutions, developed through an open-ended, trial-and-error approach and in both instances the collaborative process is still ongoing.

The paper primarily focuses on the development of the Net Work prototype and it may be that a performative approach is the only viable option when trying to design systems with even minimal agency which respond to the environment in which they are situated. This is because it is often not possible to define in advance all the significant parameters of interactive systems and their environments and consequently it is hard to predict the behaviour that will result from system-environment interactions. Penny (2000, p. 416) describes the advantages of an artistic training for building autonomous, interactive systems where the focus is on what he calls 'interactive esthetics', rather than implementing an "externally specified task for the system"; that is, the type of problem that requires a performative approach. He argues that artists are

able to experiment without the constraint of total reliability or a pragmatic work-oriented goal and consequently they can open up a wide field of possibilities, some of [which] may ultimately have application or relevance in pragmatic applications.

(Penny 2000, pp.420-421)

This insight has been confirmed in the CELL project where the collaboration between artist Jane Prophet and scientist Neil Theise led to a significant, and productive, change in his and the rest of the CELL team's approach to stem cell research. The success of this project led directly to the setting up of the IRC to investigate the benefits of applying an interdisciplinary collaborative approach to design problems.

In the next section we give a brief overview of stem cell research and some of the rather striking limitations of wet-lab experimentation that motivated the $C E L L$ project. We then describe the collaborative process of the IRC and how this led to building the Net Work prototype. This artwork was inspired by the stem cell simulations developed in the $C E L L$ project and a desire to facilitate a public understanding of the paradigm shift currently occurring in this area of biological research. As a first step towards this goal, a software implementation of Ashby's (1960) homeostat model, referred to in the opening quote in this section, is used to control the Net Work prototype. The details of this artificial neural network homeostat are given in Section 6. The paper concludes with a short summary of some of the benefits and pitfalls of interdisciplinary collaborative research that have become apparent from working on the CELL and IRC projects.

\section{Stem cell research}

Stem cells have the capacity to maintain their own numbers and also to change form - typically through cell division - in order to replenish any type of functional cell in the body (for example, blood, liver and gut cells) that continually die off in healthy living organisms as well as a result of disease 
and injury. In recent years there has been a growing debate about how stem cells behave in the human body: whether the fate of stem cells is pre-determined (Nicola and Johnson 1982) or stochastic (Thornley et al. 2003); and whether the fate of cells relies on their internal state (Novak and Stewart 1991), or on extracellular micro-environmental factors (Trentin 1970). There have been several attempts to build formal models of these theories so that predictions can be made about how and why stem cells behave both individually and collectively.

Recent experimental evidence has suggested that stem cell development may be more complicated than was originally thought. The standard model is that a stem cell becomes increasingly differentiated over time along a well-defined cell lineage and eventually becomes a fully functional cell. This model has been challenged by many researchers including one of our collaborators, Neil Theise (Theise and Krause 2002; Theise 2003). New experimental data suggests that stem cell fate is both reversible, that is cells can become less differentiated and behave more like stem cells, and plastic, that is cells can migrate from one cell lineage to another. This has led Theise and other researchers to propose new theories of stem cell development that have challenged the prevailing paradigm in biology.

Tissue slides provide the primary evidence that many medical researchers use to deduce the behaviour of living stem cells. Creating tissue slides necessitates the killing of an organism, thereby reducing a complex, dynamic system to a static, two-dimensional entity whose colour is altered through staining. The fact that stem cells cannot be observed 'in action' in the adult human body is the single most limiting issue in stem cell research. There is therefore a critical need to build simulations to understand how stem cell behaviour at a local level can produce global cellular behaviour such as the re-population of certain functional cells.

\subsection{The CELL project}

The CELL project was originally supported by the Wellcome Trust who funded artist Jane Prophet and stem cell researcher Neil Theise to discuss new theories of stem cell behaviour. As this project and the team grew, a range of approaches to investigate stem cell behaviour were employed including agent-based models, simulations, visualisations, and an art installation, Staining Space, which reflected on how different disciplines use representations and data visualisation.

Through Jane Prophet's background in ALife (for example, TechnoSphere (Prophet 2001)), the work of Mark d'Inverno in multiagent systems (d'Inverno and Luck 2004) and, moreover, through collective and sustained enquiry, Neil Theise became familiar with the notion of self-organising agent systems. The appeal to Neil Theise was that very simple rules at the local agent level could give rise to sophisticated behaviour at the system level that could be observed in simulation.

It became clear to the CELL team that the most productive way to model stem cells in the adult human body was as a dynamic system of self-organising computational agents. Simulation is particularly valuable in this field as individual stem cells cannot be identified or tracked in the adult human body. Whilst wet-lab experiments typically involve looking at slides of dead tissue, the computer provides a means to model a living, dynamic system. (Albeit it a massively simpler one than that which occurs in natural systems.) In keeping with the performative approached described in this paper, we have developed stem cell simulations using a 'suck-it-and-see' strategy, tuning the behaviour of the individual cells in the model to try to attain the desired overall system behaviour. It is perfectly possible that this latter approach may provide a key to understanding how various catastrophic illnesses such as cancer occur through the (mis)behaviour of one individual cell. 
The agent model is a behavioural one, and specifies what an individual agent will do next when it has a particular state and is in a particular environment. This metaphor has some biological plausibility and can often be more intuitive to the biologist than a mathematical model. Simulation modelling can therefore facilitate a dialogue between the wet-lab experimentalists and conceptual modellers. This process of exchange is often lacking in much conceptual or formal modelling.

The work to date has used existing, well-established techniques for specifying and modelling agent-based systems (d'Inverno et al. 2006). A major strand of the research has been an attempt to develop an agentbased model of Theise's theory of stem cell organisation in order to understand how some of the key cell-cell interactions lead to the sophisticated and robust behaviour of adult stem cell systems (Theise and d'Inverno 2003; d'Inverno and Luck 2004). Moreover, the project has been increasingly interested in the possibility that many different types of cells, in the right conditions, might be able to exhibit stem cell behaviour. If this were found to be true, it might question the notion that certain cells are stem cells per se, and this is something that would cut right across mainstream stem-cell research as we currently understand it.

What no one could have predicted at the outset of the CELL project was the massive impact that the collaboration would have on all members. For example, it led Neil Theise to radically change the conceptual framework he uses for thinking about stem-cell behaviour, moving from his practice of looking at stained 2D slides to having a clear conceptual model of dynamic interaction and self-organisation. The following text is taken from a recent paper in a biology journal by Neil Theise and is indicative of this change.

Cells fulfil all the criteria necessary to be considered agents within a complex system. they exist in great numbers; their interac- tions involve homeostatic, negative feedback loops; and they respond to local environmental cues with limited stochasticity (quenched disorder). Like any group of interacting individuals fulfilling these criteria, they selforganize without external planning. What emerges is the structure and function of our tissues, organs and bodies.

(Theise 2005)

The experiences of the $C E L L$ project motivated Jane Prophet and Mark d'Inverno to set up a research cluster to further investigate the potential of interdisciplinary research especially in the context of agent-based and interactive systems in design.

\section{Interdisciplinary Research Cluster (IRC)}

Motivated by the experience of using selforganising agent-based approaches to facilitate new understandings of biological systems, and keen to explore the potential benefits of interdisciplinary collaboration further, Mark d'Inverno and Jane Prophet set up an Interdisciplinary Research Cluster (IRC) funded by the EPSRC and AHRC entitled: 'Designing physical artefacts from computational simulations and building computational simulations of physical systems' (www.interdisciplinary. co.uk). The major aim of this project was to form a new research community centred on simulation and digital art and design. Specifically, we are interested in exploring how interdisciplinary collaboration can lead to new forms of design suitable for the challenges of the $21^{\text {st }}$ century.

\subsection{IRC methodology}

Even though there was some notion of the kinds of issues we wanted to explore in the IRC, we had no strict plans or schedules that told us how we should go about our activity. Despite setting up a newsgroup and website, and putting out various calls to join our community, the traffic on the website was 
initially very low. However, we did organise several meetings of a very heterogeneous set of people (including artists, designers, computer scientists and engineers) and decided that in order to explore the issues it would be best to actually build a physical artefact that had computational and generative elements. Ten projects were proposed by individuals or teams and investigated over a 2-day workshop. Towards the end of the workshop a vote was taken to make a prototype of Net Work, Jane Prophet's proposed interactive installation.

Because members of our group felt a sense of ownership, not only of the goal to collectively build a physical artefact, but also of the design process, the traffic on the newsgroup increased significantly. Many members of the cluster gave their time freely to argue and consider the best way to build the Net Work prototype. The advantage of having a group of collaborators, with a broad range of artistic, computing and engineering skills was evidenced by the large number of potential solutions offered to software and engineering problems, the subsequent high level debate, and the speed at which the research cluster

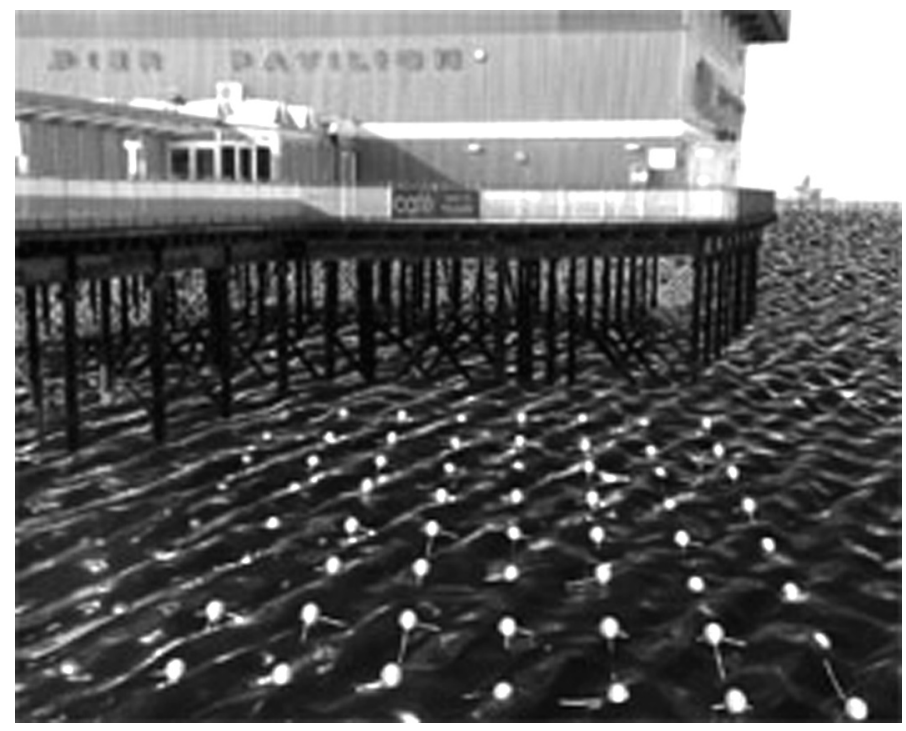

Figure 1. A visualisation of Net Work adjacent to the pier in Herne Bay, UK. solved many of the challenges associated with building Net Work. The distributed collaboration prompted the lead artist Jane Prophet to further develop and clarify her core idea and the project evolved as collaborators introduced and argued for new elements. Having built a prototype, there are still several outstanding technical challenges to be solved and the design will evolve further through collaboration.

\section{$4 \quad$ Net Work}

Net Work will be a large scale interactive art installation in Herne Bay, UK, constructed initially from autonomous buoys placed at 1 metre intervals to form a $10 \times 10$ grid (Figure 1). Ultimately our vision is to create a larger 50 x 50 grid of 2500 buoys. Each buoy emits different coloured light in response to the movement of the water detected by tilt sensors, light levels affected by users shining torches and the state of neighbouring cells.

As mentioned in section 2.1, one of the outcomes of the CELL project was an art installation, Staining Space, which although successful in reflecting on the way different disciplines visualise data, did not contribute significantly to the public understanding of how living cells behave. The models of stemcell behaviour built by the CELL team were based on very simple rules specifying cell-cell interaction and how cells react to environmental changes. Descriptions of these simple rules were posted on the cluster's website and Jon Bird, one of the cluster's original members, proposed using a very simple homeostat model to drive the activity of the autonomous buoys.

This cybernetic model, first proposed by Ashby (1960), might provide first steps in the public understanding of self-organising processes, which in turn could lead to insights into more complex systems such as stem cells. To further facilitate this understanding, Net Work will provide two types of interaction to enable 
users to engage with the artwork and affect its behaviour: the local audience can shine torchlight on the buoys to activate their light sensors; and a remote audience can affect the work over the Internet. In both cases the state of a buoy is changed and they will respond by emitting different colours. Using a homeostat control system is also appropriate because it is illustrative of the performative approach to problem solving, as Pickering (2002) notes in the quote at the beginning of the paper. The next two sections describe Ashby's homeostat model and how it is implemented in Net Work.

\section{$5 \quad$ Homeostasis}

Canon (1939) coined the term 'homeostasis' to refer to the self-regulating mechanisms of an organism that enable it to maintain its essential physiological variables, such as body temperature and blood pressure, in a state of dynamic balance. Cybernetics proposed that self-correcting (negative) feedback loops constitute the pattern of organization that underlies homeostasis and adaptive behaviour in organisms (Rosenbleuth et al. 1943). A text book example of a negative feedback system is a thermostat, which adjusts the output of a heating system to keep the temperature in a room at a specified value.

Ashby (1960) extended the cybernetic concept of a self-correcting system and investigated both theoretically and empirically the organization of more complex self-regulating systems that can adapt to radical disruptions. He was motivated by the question: what sort of thermostat could, if assembled at random, rearrange its own parts to get itself stable for temperature?

(Ashby 1960, p.70).

He defined an ultrastable system as one that is able to reconfigure plastically in response to any of its essential variables going outside their stable bounds and thereby return the variable to an acceptable level. Ashby argued that in order for ultrastable mechanisms to adapt in this way they necessarily consist of both a primary feedback between the sensorimotor system and the environment and a secondary, intermittent feedback between the essential variables and the sensorimotor system. The secondary feedback reconfigures the primary feedback connections when the essential variables go outside of given limits.

\subsection{Ashby's homeostat}

Ashby built the homeostat in order to empirically demonstrate his theoretical arguments about ultrastable mechanisms. The homeostat (Figure 2) is comprised of units, each of which consists of a magnet, electronic circuitry and other physical components. The magnetic field drives the position of a needle on top of the unit which is free to move in an approximately 180 degree arc. The needles' positions represent the essential variables of the system that are to be kept within bounds, which are defined as 45 degrees either side of a central point at the front of each unit.

All four units are connected to each other electrically and the torque on each magnet is proportional to the sum of the three input currents from the neighbouring units and a single recurrent connection. The current that a unit sends to its neighbours, and feeds back to itself, is proportional to the deviation of its needle from the central position. No current is passed if the needle is positioned within the stable region and larger currents are passed as the deviation from this region increases. This relationship is implemented by the needle dipping into a trough of water placed below it. A constant voltage is passed across the trough and the needle picks up a potential which is dependent on its position relative to the water. The viscosity of the water affects the stability of the homeostat: a higher viscosity dampens the movement of the needle and makes the system more stable; lower viscosity leads to more fluctuating, dynamic behaviour.

Each of the units can be arbitrarily conceptualised as representing the environment 


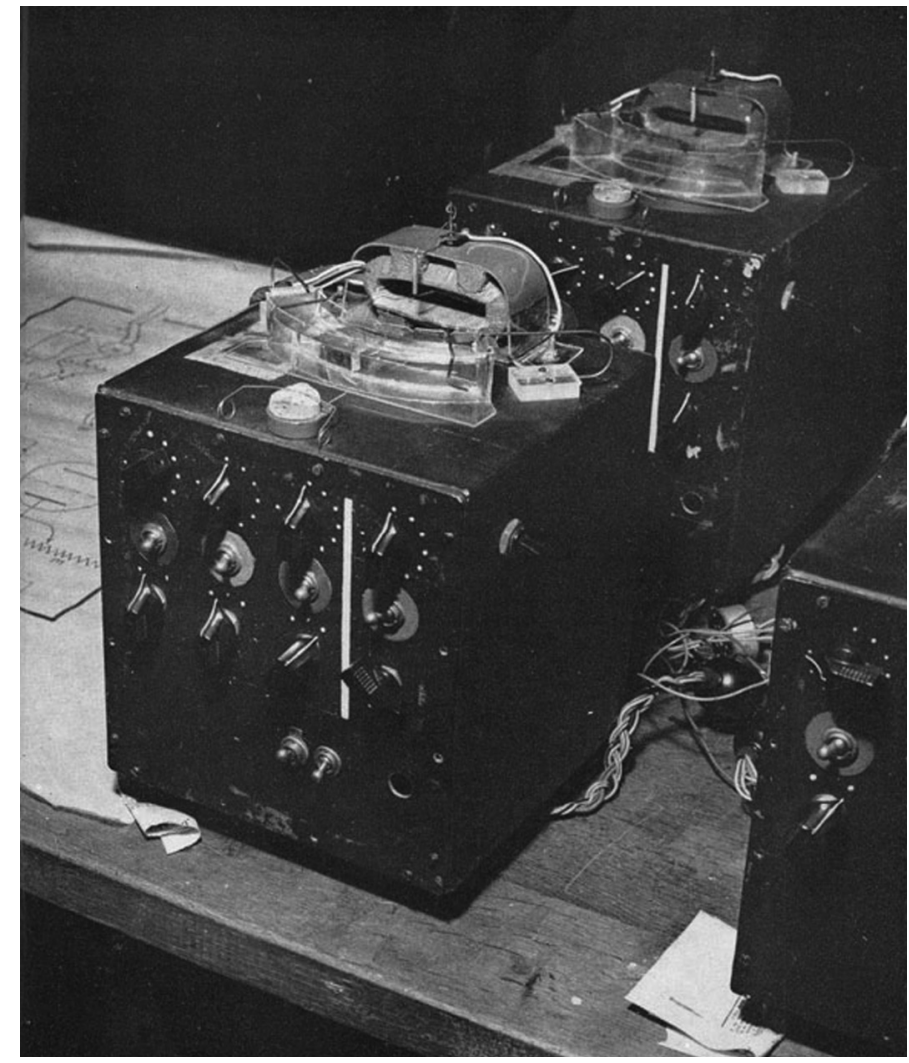

Figure 2. Ashby's four unit homeostat.

or sensorimotor system of an ultrastable mechanism and the electrical interactions between the units therefore model the primary feedback. When a needle deviates outside of its stable bounds, a secondary feedback mechanism is triggered that randomly changes a number of parameters that affect the movement of the magnet. The magnet in each unit is driven by the activity of four coils, each of which is dependent on the settings of an associated commutator and potentiometer. Three of the coils are connected to one of the input connections from a neighbouring unit and the other coil is connected to the recurrent connection. The polarity of each input, including the self-connection, is determined by the state of the commutator and the proportion of each input signal reaching its associated coil is determined by the state of the potentiometer. The secondary feedback is implemented by connecting a uniselector to each unit. This device has 25 discrete states, each of which consists of a triple of random values, derived from a standard statistical table. This can be thought of as a look-up table with 25 rows and 3 columns which provides random numbers to reconfigure the system; in contemporary digital systems a pseudo-random number generator plays a similar functional role. Each of these random values controls the operation of one of the commutator/potentiometer pairs and thereby determines the weighting and polarity of an input connection from a neighbouring unit. There are $25^{4}(390,625)$ different combinations of uniselector parameter values that a four unit homeostat can randomly explore in order to find a combination that leads to all of the units displaying stable behaviour. When each potentiometer/commutator pair is assigned a new random parameter value, the polarity and amplitude of the associated input current are changed. This affects the movement of a unit's needle and in turn, through its electrical connections, the movement of the needles in the other units of the homeostat. Through this simple, random mechanism the homeostat behaves as though it were seeking to keep its needles in central positions in a goal-oriented fashion.

Ashby tested the homeostat by first allowing it to stabilise and then taking control of one of the units and reversing the commutator by hand, thereby causing an instability. He then observed how the system adapted its configuration until it found a stable state once more. In his initial experiments he used a simple two unit system, where unit $M 1$ was controlled by hand and unit $M 2$ was controlled by its uniselector. When Ashby reversed the commutator by hand, he found that only a relatively small number of uniselector changes in $M 2$ were required for stability to be restored. Ashby also investigated the response of a 3 unit homeostat to perturbation, where 
unit $M 1$ was connected to $M 2$ and uniselector controlled, $M 2$ was connected to $M 3$ and hand controlled, and $M 3$ was connected to $M 1$ and was fixed so that the movement of M3 moved M1 in the opposite direction. Ashby again found that only a small number of uniselector changes in unit were required before the system was stable. He argued that the homeostat displayed,

in elementary form, the power of self-organisation

that was analogous to the action of the nervous system:

first the established reaction, then an alteration made in the environment by the experimenter, and finally a reorganisation within the nervous system, compensating for the experimental alteration. (Ashby 1960, p.107).

Recently, there has been a renewed interest in the homeostat and there have been several different applications of ultrastable architectures by artificial life researchers. Di Paolo (2000; 2003) carried out a number of experiments that explore the link between homeostasis and adaptive behaviour using simulated robots that perform phototaxis. He confirmed that a homeostat architecture can adapt to radical sensorimotor disruptions, namely the reversal of the connections between light sensors and the robot control system. Eldridge (2002) implemented neural network simulations of the homeostat and used them as a component in number of generative music systems. An excerpt from one of her musical compositions, Fond Punctions, can be downloaded from: http://www.ecila.org. Her neural network model is the basis of the implementation used in Net Work and is discussed in detail in Section 6.2. Eldridge's model was able to functionally replicate the behaviour of Ashby's four unit homeostat, stabilising after both minor and major perturbations to the activity of units in the network. Eldridge found that there were three significant factors that affected the time it took the system to adapt to the activation of any of the neurons going outside their stable bounds:

1 changing the viscosity parameter: the larger the value, the more damping on the system and consequently the more stable the network;

2 increasing the number of units: the larger the number, the longer the system took to stabilise;

3 changing the degree of connectivity: the more interconnections between units, the longer the system took to stabilise.

\section{The homeostat model in Net Work}

The homeostat simulation used to drive the Net Work prototype was based on Eldridge's (2002) neural network $C$ code, although different parameter values were used. It was re-implemented in Java in the Processing environment (http://processing.org). The user interface was built using John Beech's $M y G U I$ library.

The source code and interactive demonstrations of the homeostat model used in the Net Work project are online at: www.cogs. susx.ac.uk/users/jonba/homeostat/instructions. htm

\subsection{Visualizing the homeostat activation dynamics}

In the simulation each buoy is represented by a neural network unit. In order to visualize the dynamics of the network the activation of each unit is mapped to a hue value (using the HSB colour model). The hue representing the stable activity was empirically determined so that changes in the activation could be clearly visualized. For example, shifting the stable hue to either the green or red regions of the HSB colour space makes it more difficult to visualize small changes in activity. Currently only the hue of the units is used to visualize changes in activation and saturation and brightness stay constant at 0 . There are two 
approaches to dealing with hue values at the extreme of the range: either the activation/hue can be limited to the minimum or maximum value or the activation/hue can go 'around the colour wheel', that is:

If hue $>=$ maximum hue (1.0) then hue $=$ hue - maximum hue;

If hue $<=$ minimum hue $(0)$ then hue $=$ maximum hue + hue .

The simulation allows both options, but currently we are using the 'round the wheel' method, which is appropriate for the HSB colour model where hue values are adjacent on the colour wheel. Whereas the needle in the homeostat was restricted to moving in a 180 degree arc, in our system the analogue of the needle can rotate through 360 degrees.

\subsection{The homeostatic network}

In the Net Work simulation each unit is connected to its four nearest neighbours (positioned N, E, S and W). The number of connections was restricted in order to minimise the time it takes the network to stabilize. The activation of each of the units is in the range $[-1.0,1.0]$ and their stable range is $[-0.1,0.1]$. The sign of the weights represents the action of the commutator and the strength the action of the potentiometer in Ashby's original homeostat. The weight of the recurrent connection is fixed at -0.01 as this helps to quickly stabilize the activity of the units. If a unit's activity goes outside of its stable bounds then the sign and strength of its four connections to its nearest neighbours (but not its recurrent connection) are randomly changed by setting each connection to a different random value in the range $[-1.0,1.0]$. The maximum change in activity of a unit in a single time step is initially set to \pm 0.01 , but this value can be changed in the user interface (this corresponds to the viscosity of the liquid in the homeostat). Each time the homeostatic network is updated a small noise value is added to the activation of each of the units (range [0,
0.03 ], but this value can also be changed in the user interface). Initially, the activation of each of the units is set to a random value in the range $[-0.03,0.03]$.

Each unit in the network is governed by the following equation:

$$
z_{i}^{t+1}=z_{i}^{t}+\delta_{\max } \sum_{j=1}^{5} w_{i j} z_{j}^{t}
$$

where $z_{i}^{t+1}$ is the activation of neuron $z_{i}$ at time step $t+1$ in the simulation, $w_{i j}$ is the connection weight from unit $z_{j}$ to unit $z_{i}$ and $\delta_{\max }$ is a damping factor that limits the maximum change in unit activation and is the analogue of the viscosity in Ashby's homeostat. Equation 1 shows the update rule for a unit with 5 connections (1 recurrent connection and 4 connections to its nearest neighbours) but the simulation enables a user to increase the number of connections that each unit makes to neighbouring units.

\subsection{Designing the interaction}

By making the network sensitive to the motion of waves, it will hopefully become evident to observers that the buoys are connected in a network, rather than independent units. In the simulation a wave is passed under the network of buoys from left to right. When a wave passes under a simulated buoy, its activation is increased by 0.2 , causing its activity to pass outside of the stable bounds. This in turn leads to a reconfiguration of the units' connections. In the final physical installation it will be possible to correlate the network dynamics with the choppiness of the sea: the calmer it is, the lower the noise level and smaller the maximum unit change; the rougher the sea, the higher the noise level and larger the maximum unit change.

The simulation can run in three different modes: random, where the activation of the units and their corresponding hue is randomly changed at each update; homeostatic, where the network weights reconfigure if a 
unit's activation goes outside of its stable bounds; and dual mode, where the network alternates between random and homeostatic modes. In dual mode the activity of the units is randomly changed until the variance of the activation reaches a given threshold, at which point the homeostatic mechanism kicks in, reconfiguring the network and making it increasingly stable until the variance of the activation of the units has gone below a lower threshold. When this lower threshold is crossed, the homeostatic network is turned off and the activation of the units in the network is randomly changed once more.

\subsection{Open questions about Net Work}

Some open questions that are still being explored in the project are: Can an observer of the hue dynamics infer anything about the underlying system? Does Net Work's dynamic responses to wave action give the sense that the system has 'minimal agency'? Does the current homeostat control system facilitate the public understanding of self-organising systems and will this in turn lead to a wider understanding of stem-cell behaviour? It will be only through a trial-and-error process that we can determine whether a homeostatic network is a useful mechanism for achieving this and more generally, how viewers perceive the activity of Net Work. A question closely related to these issues is: How can a viewer be encouraged to explore the work? Currently, in the simulation when a user clicks on a unit, its hue is set to a light blue colour $(0.2+$ a random value in the range $[0,0.1])$. This colour persists for 4 seconds before the homeostatic mechanism kicks in and stabilises the unit's activation. The system is therefore generating a static response whereas a more engaging system would behave in such a way that the user's future responses are influenced. A perhaps more effective approach, appropriate to the

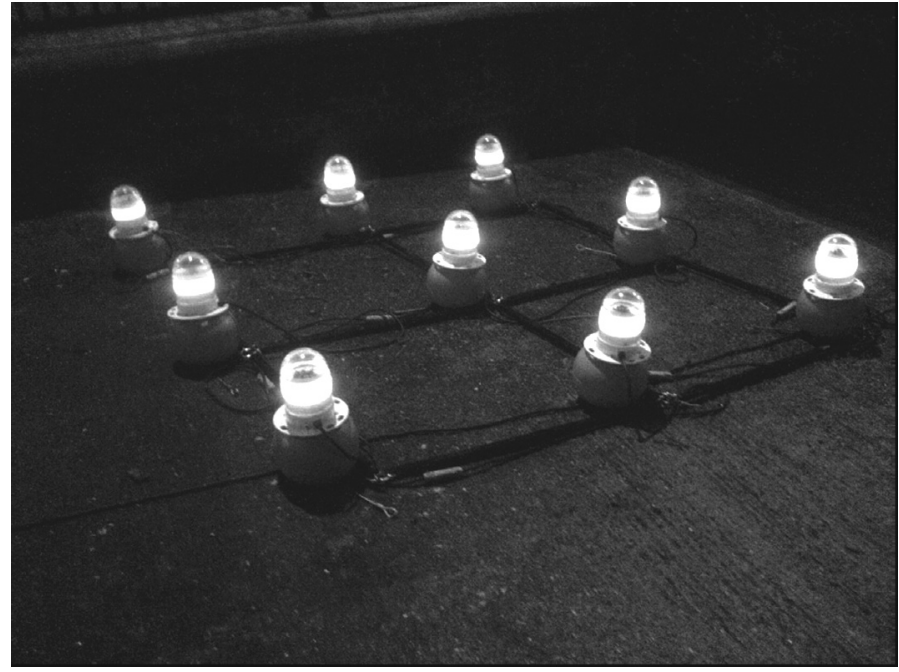

Figure 3. A $3 \times 3$ grid prototype of Net Work prior to testing in the Thames.

site, would be for users' torch beams to act like stones thrown into the network causing ripples of activity to pass through the system which would in turn interact with the action of the waves. The two sorts of stimulation (wave- and user-induced) could have distinguishing hues. The Net Work prototype has resolved many of the challenging technical issues involved in building an interactive artwork that floats in the sea. However, it will require further exploration to fine tune the interaction processes and control system of the artwork and to ensure that the installation responds effectively to its site by the pier in Herne Bay (Figure 1).

\section{Discussion}

Even with a relatively simple system such as Net Work, there are a large number of parameters that affect its behaviour, and in order to discover the appropriate parameter ranges it is important to explore the effects of different values. We implemented a simple interface to enable experimentation with some system 
parameters in simulation in order to explore how different values affect the behaviour of the system. For example, it became evident through building the homeostatic network that the range of stable activation must be close to zero. Some experiments were carried out to see whether a homeostat could stabilize its unit activations to positive or negative values and it was found that with the current network architecture and parameter ranges this was not possible.

In the future, we wish to implement a large scale Net Work and it will depend on raising relatively large amounts of funding. Successful funding will result not only in the production and exhibition of Net Work, but will include more collaborative problem solving of outstanding design challenges, such as wireless communication between buoys and the use of solar power. It is also critical that the interaction between users and $\mathrm{Net}$ Work facilitates an understanding of the selforganising capacity of the homeostat model or more complex algorithms that might be implemented on the system. A $3 \times 3$ prototype has shown that many of the technical issues can be solved (Figure 3). However, more issues will only become apparent when a larger network of buoys is constructed in situ at Herne Bay.

\subsection{Reflections on interdisciplinary collaboration}

Many participants in the IRC spoke about their experience of interdisciplinary collaborations and it is worth reflecting on the opportunities and difficulties that were reported. Clearly, on the positive side, the pooling of knowledge, especially when people have different expertise and experience, can speed up problem solving. Discussion around central topics and sharing prior experience often results in 'added value', namely, problems encountered in other projects by collaborators can be solved as a side effect of focusing on the core challenges in a named design. In explaining our 'common knowledge' to those of another discipline insights can be gained and stand points previously 'taken as read' can be usefully challenged. Large group collaborations that are carefully structured early on so that each member has the opportunity to give position papers and to 'show and tell' can lead to numerous collaborations between members that continue beyond the life of the project that members came together to work on.

On the down-side, interdisciplinary research can often be seen as 'soft' or 'flaky' by researchers working in single discipline areas. There are neither journals nor clearly defined peer-review procedures for ensuring and demonstrating quality in interdisciplinary research and funding for collaborative interdisciplinary research is very limited. Consequently, researchers may lose interest and withdraw from interdisciplinary projects or not be attracted to working on them at all. Moreover, there is a leap of faith that has to occur when one starts to engage in such a project. There are often no clear goals, and it takes a lot of negotiation before any substantial progress can be made - this is inherent in the performative approach. It takes time and patience to build the trust necessary for a collaboration to be most beneficial. Collaborators can be inflexible and insist that their way is the best or find it difficult to balance the priorities of different disciplines (for example, that an art venue and audience may not find 'illustrated science' engaging). Consequently, there often need to be lengthy explanations to collaborators from other disciplines, which can be both time consuming and taxing.

Clearly, many serious issues need to be addressed in the $21^{\text {st }}$ century. For example, global warming, urbanisation, immigration and terrorism. It is evident that there are problems in these areas but they are not well defined and we believe it will require 
an interdisciplinary performative approach if they are to be solved. Even though our efforts are tiny in comparison we hope they have raised some issues which will also be relevant to larger scale projects in a number of different areas.

\section{Acknowledgements}

We would like to thank all members of the IRC that were involved in the development of the Net Work prototype especially Michael Bacon, Adrian Bowyer, Trevor Graham, Paul Hammond, Jon McCormack, Luke Nicholson, Fredrik Olofsson, Ben Pirt, Rob Saunders, Neil Theise and all members of the interdisciplinary research cluster.

Jane Prophet and Mark d'Inverno would like to thank their collaborators on the CELL project: Peter Ride, Neil Theise and Rob Saunders.

\section{References}

Ashby, W. R. (1960) Design for a brain: the origin of adaptive behaviour, second edition, Chapman and Hall, London.

Austin, J. L. (1962) How to do things with words, Oxford University Press.

Canon, W. B. (1939) The wisdom of the body, Norton, London.

Di Paolo, E. A. (2000) 'Homeostatic adaptation to inversion of the visual field and other sensorimotor disruptions' in From animals to animats 6: Proceedings of the 6th International Conference on the Simulation of Adaptive Behavior, eds J.-A Meyer, A. Berthoz, D. Floreano, H. Roitblat and S. Wilson, MIT Press, Cambridge, MA, pp. 440-449.

Di Paolo, E. A. (2003) 'Organismically-inspired robotics: homeostatic adaptation and natural teleology beyond the closed sensorimotor loop' in Dynamical systems approach to embodiment and sociality, eds K. Murase and T. Asakura, Advanced Knowledge International, Adelaide, Australia, pp. 19-42.

d'Inverno, M. and Luck, M. (2004) Understanding agent systems, second edition, Springer.

d'Inverno, M., Theise, N. D. and Prophet, J. (2006) 'Mathematical modelling of stem cells: a complexity primer for the stem cell biologist' in Tissue stem cells: biology and applications, eds C. Potten, J. Watson, R. Clarke and A. Renehan, Marcel Dekker, pp. 1-47.

Eldridge, A. (2002) 'Adaptive systems music: algorithmic process as a compositional tool', MSc, University of Sussex, [online], http://www. informatics.sussex.ac.uk/users/alicee/NEWSITE/ ecila_files/content/papers/ACEMScThesis.pdf.

Nicola, N. and Johnson, G. (1982) 'The production of committed hemopoietic colony-forming cells from multipotential precursor cells in vitro', Blood, vol. 60, pp. 1019-1029.

J. Novak and C. Stewart (1991) 'Stochastic versus deterministic in haemopoiesis: what is what?'. $\mathrm{Br}$ J Haematol, vol. 60, pp. 527-529.

Penny, S. (2000) 'Agents as artworks and agent design as artistic practice' in Human cognition and social agent technology, ed. K. Dautenhahn, John Benjamins, Amsterdam, pp. 395-414.

Pickering, A. (2002) 'Cybernetics and the mangle: Ashby, beer and pask', Social Studies of Science, vol. 32, pp. 413-437.

Prophet, J. (2001) 'Technosphere: "real" time "artificial" life', Leonardo: The Journal of the International Society for The Arts, Sciences and Technology, vol. 34, no. 4, pp. 309-312.

Rosenbleuth, A., Wiener, N. and Bigelow, J. (1943) 'Behavior, purpose and teleology', Philosophy of Science, vol. 10, pp. 18-43.

Schlieben, K. (2002) Performative curating, [online], http://www.kunstverein-muenchen.de/

Theise, N. D. (2003) 'New principles of cell plasticity', C R Biologies, no. 325, pp. 1039-1043.

Theise, N. D. (2005) 'Now you see it now you don't', Nature.

Theise, N. D. and d'Inverno, M. (2003) 'Understanding cell lineages as complex adaptive 
systems', Blood, Cells, Molecules and Diseases, vol. 32, pp. 17-20.

Theise, N. D. and Krause, D. S. (2002) 'Toward a new paradigm of cell plasticity', Leukemia, vol. 16 , pp. 542-548.

Thornley, I., Sutherland, R., Wynn, R., Nayar, R., Sung, L., Corpus, G., Kiss, T., Lipton, J., Doyle, F., Saunders, J., Kamel-Reid, S., Freedman, M. and Messner, H. (2003) 'Early hematopoietic reconstitution after clinical stem cell transplantation: evidence for stochastic stem cell behavior and limited acceleration in telomere loss', Blood, vol. 99, pp. 2837-96.

Trentin, J. (1970) 'Influence of hematopoietic organ stroma (hematopoieticinductive microenvironment) on stem cell differentiation' in Regulation of hematopoiesis, Volume 1, ed. A.S. Gordon, Appleton-Century-Crofts, New York, pp. 161-168.

Jon Bird is a Research Fellow at the University of Sussex where he models adaptive behaviour using computer simulations and robots. Currently he is working on the DrawBots project that aims to artificially evolve drawing robots. Applying his research across disciplines he has published several papers that simultaneously consider both the artistic and scientific potential of emergent and evolutionary systems. He is the co-founder of Blip, a Brighton-based arts-science forum.
Mark d'Inverno gained an MA in Mathematics and an MSc in Computation at the University of Oxford, and a $\mathrm{PhD}$ from University College London. He is currently Professor of Computer Science at Goldsmiths College, University of London. His interests focus on intelligent agent systems and encompass many aspects of agency including action, perception, deliberation, communication, negotiation and social norms. In recent years he has applied his theoretical agent-based work in more interdisciplinary settings such as biological modeling, computer-generated music, art and design. His main interest is in understanding how creativity and innovation can occur and be exploited at the interface of art, design, science and technology.

Jane Prophet is a British artist. Her projects include Conductor (made by flooding a power station with 74 tonnes of water and lighting it with 120 electro-luminescent cables). Decoy and The Landscape Room combine images of real and computer simulated landscapes. Her latest work, Model Landscapes, uses rapid prototyping technology to make miniature trees from mathematical data and display them using tiny video screens. She recently completed a NESTA 'Dream Time' Fellowship and is currently developing her interdisciplinary collaborations, in particular Net Work (hundreds of illuminated buoys) and Big Plastic Tree (automated artwork built by robots). 